\title{
The Path Integral Monte Carlo Method for Quantum Transport on a Parallel Computer
}

\author{
C. Schulz-Mirbach \\ Arbeitsbereich Hochfrequenztechnik \\ Technische Universität Hamburg-Harburg \\ Wailgraben 55, D-21071 Hamburg, Germany
}

\begin{abstract}
Based on the Feynman path integral formulation for the time evolution amplitude, we compute the quantum mechanical transition probability for a charge carrier in a semiconductor crystal. Our implementation is performed on a parallel computer (Parsytec GC 64). We discuss the ability of the method to achieve a spatially resolved probability amplitude which is necessary for the analysis of quantum electronic devices. Macroscopic observables are evaluated using this probability function. It complements the conventional distribution function which results from the solution of the semi-classical Boltzmann transport equation.
\end{abstract}

\section{Introduction}

It has been suggested that the Feynman path integral formulation of quantum mechanics is an appropriate method for describing quantum transport in ultrasmall electronic devices [1], [2]. One of the advantages of the method is that the coupling of charge carriers to phonons and other scattering processes, non-constant electric fields which are not necessarily sinusoidal, as well as complicated band and quantum well structures can easily be included, which are encountered in hetero-structure devices such as Resonant Tunneling Diodes. Thanks to these promising facts and despite of the drawbacks of the method like high CPU time requirements and the difficulty to estimate the error of the approximations, we aim to derive a simulation technique which is intended to overcome the limitations of the Boltzmann transport equation. Due to the wave-like character of the charge carriers, there is a limit in the characteristic length of devices above which carriers can be described as localized particles. Hetero-structure devices are an example for this situation.

\section{Path integrals on a discretized time axis}

The probability amplitude of a physical system to evolve from time $t_{a}$ to $t_{b}$ is given by the matrix elements of the time-evolution operator $U\left(t_{b}, t_{a}\right)=\exp \left(i \mathcal{H}\left(t_{b}-t_{a}\right) / h\right)$, where $\mathcal{H}$ is the Hamiltonian of the system [3]. In order to study the momentum 
distribution function for homogeneous material it is appropriate to expand $U\left(t_{b}, t_{a}\right)$ in momentum eigenfunctions, where the following expression evolves ${ }^{1}$

$$
\begin{aligned}
& \left(\vec{p}_{b}, t_{b} \mid \vec{p}_{a}, t_{a}\right)=<\vec{p}_{b}\left|U\left(t_{b}, t_{a}\right)\right| \vec{p}_{a}> \\
& \quad \approx \prod_{n=1}^{N}\left[\int_{-\infty}^{\infty} \frac{d \vec{p}_{n}}{2 \pi \hbar}\right] \prod_{n=1}^{N+1}\left[\int_{-\infty}^{\infty} d \vec{r}_{n}\right] \\
& \quad \times \exp \left\{\frac{i}{\hbar} \sum_{n=1}^{N+1}\left[-\vec{r}_{n}\left(\vec{p}_{n}-\vec{p}_{n-1}\right)-\frac{\left(t_{b}-t_{a}\right)}{N+1} \mathcal{H}\left(\vec{p}_{n}, \vec{r}_{n}, t_{n}\right)\right]\right\} \\
& \quad t_{0}=t_{a}, \quad t_{N+1}=t_{b}
\end{aligned}
$$

This expression means that the exponential has to be evaluated for all momentumspace paths $\vec{p}_{a} \sim>\vec{p}_{b}$ with the resulting terms being summed up. The spatial integrals refer to time points $t_{1} \ldots t_{N+1}$, so all possible spatial end points are summed up. No spatial point $\vec{r}_{0}$ occurs. ${ }^{2}$

The momentum time evolution amplitude $\left(\vec{p}_{b}, t_{b} \mid \vec{p}_{a}, t_{a}\right)$ equals the conditional probability amplitude for a particle to come to a momentum state $\mid \vec{p}_{b}>$ at time $t_{b}$ after starting with $\left|\vec{p}_{a}\right\rangle$ at time $t_{a}$. Assuming knowledge of the wavefunction $\Psi\left(\vec{p}, t_{a}\right)$, the probability of measuring a momentum $\vec{p}$ at time $t_{b}$ is given by the distribution function $f\left(\vec{p}, t_{b}\right)$

$$
f\left(\vec{p}, t_{b}\right)=\left|\Psi\left(\vec{p}_{b}, t_{b}\right)\right|^{2}=\left|\int \frac{d \vec{p}_{a}}{(2 \pi \hbar)^{3}}\left(\vec{p}_{b}, t_{b} \mid \vec{p}_{a}, t_{a}\right) \Psi\left(\vec{p}_{a}, t_{a}\right)\right|^{2}
$$

Defining cells at time $t_{a}$ around the momentum values $\left\{\vec{p}_{a}^{1}, \vec{p}_{a}^{2}, \vec{p}_{a}^{3}, \ldots\right\}$ and at time $t_{b},\left\{\vec{p}_{b}^{1}, \vec{p}_{b}^{2}, \vec{p}_{b}^{3}, \ldots\right\}$, the following calculation scheme is set up

$$
\begin{aligned}
& f\left(\vec{p}_{b}^{1}, t_{b}\right)=\left|\left(\vec{p}_{b}^{1}, t_{b} \mid \vec{p}_{a}^{1}, t_{a}\right)+\left(\vec{p}_{b}^{1}, t_{b} \mid \vec{p}_{a}^{2}, t_{a}\right)+\ldots\right|^{2} \\
& f\left(\vec{p}_{b}^{2}, t_{b}\right)=\left|\left(\vec{p}_{b}^{2}, t_{b} \mid \vec{p}_{a}^{1}, t_{a}\right)+\left(\vec{p}_{b}^{2}, t_{b} \mid \vec{p}_{a}^{2}, t_{a}\right)+\ldots\right|^{2}
\end{aligned}
$$

In our calculations we assume

$$
\Psi\left(\vec{p}_{a}, t_{a}\right)=<\vec{p}_{a} \mid \Psi\left(t_{a}\right)>=\sum_{i}^{M}(2 \pi \hbar)^{3} \delta\left(\vec{p}_{a}-\vec{p}_{i}^{M B}\right) / \sqrt{M},
$$

where the $\vec{p}_{i}^{M B}$ are drawn from a Maxwell-Boltzmann-distribution. The consequence of the $\delta$-function is, that one has to consider only momentum values $\vec{p}_{i}^{M B}$ as path beginning values. It is important to mention that by the same way any momentum distribution function resulting from semiclassical methods (e.g. Monte Carlo solution of the Boltzmann transport equation) could be used. That is a possible way of combining semiclassical and quantum regime transport.

The Hamiltonian includes an imaginary potential term for the scattering of the charge carriers and the energy term due to the electric field as well as a term describing the conduction band structure. The imaginary potential term leads to a factor $\exp \left(-\sum_{n=1}^{N+1} / 2 \tau\left(\vec{p}_{n}\right)\right)$ in the integral, stochastically damping out momentum configurations being associated with high scattering rates $1 / \tau(\vec{p})$. Thus the momentum

\footnotetext{
${ }^{1}$ The equality holds only for $N \rightarrow \infty$. In the numerical treatment $N$ has to be finite.

${ }^{2}$ The Dirac bra-ket notation is used. The subindex of the eigenfunctions $\left|\vec{p}_{n}\right\rangle$ denotes them belonging to the corresponding time point $t_{n}$.
} 
integrals can be performed using the Metropolis algorithm (cf. [4]). The space integrals are reduced to integrals over boxes acompanying the classical space path. The box size is chosen to be of range of the characteristic length, which is considered to be a measure for the size of the quantum mechanical fluctuations. This method corresponds to the windowing technique described in [2]. From the point of view of quantum device simulations, the interesting regions are those where no classical paths exist. The estimate for the size of the integration boxes is of crucial importance.

The momentum axis lying in the direction of the electric field is subdivided into cells. For each path ending in one of the cells, the corresponging exponential is summed up by its real and imaginary part separately, yielding the quantum mechanical phase.

\section{Spatially resolved distribution functions for quantum devices}

For the understanding of the quantum interference phenomena in heterostructures one is interested in a spatially resolved momentum distribution function. By expanding the time evolution operator in space eigenfunctions yields the more familiar path integral

$$
\begin{aligned}
&\left(\vec{r}_{b}, t_{b} \mid \vec{r}_{a}, t_{a}\right) \approx \prod_{n=1}^{N}\left[\int_{-\infty}^{\infty} d \vec{r}_{n}\right] \prod_{n=1}^{N+1}\left[\int_{-\infty}^{\infty} \frac{d \vec{p}_{n}}{2 \pi \hbar}\right] \\
& \quad \times \exp \left\{\frac{\imath}{\hbar} \sum_{n=1}^{N+1}\left[\vec{p}_{n}\left(\vec{r}_{n}-\vec{r}_{n-1}\right)-\frac{\left(t_{b}-t_{a}\right)}{N+1} \mathcal{H}\left(\vec{r}_{n}, \vec{p}_{n}, t_{n}\right)\right]\right\} .
\end{aligned}
$$

In this expression the initial position $\vec{r}_{0}=\vec{r}_{b}$ is assumed to be given. By defining space cells (in the direction of the electric field) $x^{\text {cell } 1}, x^{\text {cell } 2}, \ldots$ for the end point of time the probability of reaching a cell and the velocity $v$ equals

$$
\operatorname{Prob}(\text { cell } i)=\left|\left(x^{\text {cell } i}, t_{b} \mid x_{a}, t_{a}\right)\right|^{2}, \quad v=\frac{\left|\left(x^{\text {cell } i}, t_{b} \mid x_{a}, t_{a}\right)\right|^{2} \frac{\left(x^{\text {cell }_{2}}-x_{a}\right)}{t_{b}-t_{a}}}{\sum_{i}\left|\left(x^{\text {cell } i}, t_{b} \mid x_{a}, t_{a}\right)\right|^{2}} .
$$

For each spatial cell we define momentum cells and sum up the probabilities for the momentum occurring in it. This is how to get a spatially resolved momentum distribution function, e.g. to analyse the region behind the barriers in a DoubleBarrier-RTD. ${ }^{3}$

\section{Importance Sampling on the Parallel Computer}

Our implementations are performed on a parallel computer containing 64 nodes with two Power PC plus processors each (80 MFlops, $16 \mathrm{MB}$ memory per node). A unit of 16 nodes is connected in a fixed topology with the other units. We use the Parix runtime-system with the programming language $\mathrm{C}$. We define virtual link connections from one node to all the others, thus obtaining a farming model [5]. The farmer organizes the distribution of the starting data and the collection of the results.

The program calculates the momentum distribution function for a fixed time interval. Every processor generates one chain of paths, thus the communication between the

\footnotetext{
${ }^{3}$ The momentum distribution for a spatial region contains less phase information than the one derived above, since the contributing paths are taken from a small spatial region. The interference with paths ending in other regions is not accounted for in this sum.
} 
nodes is kept to a minimum. In order to save memory, the momentum distribution was calculated only in the direction of the electric field. Two other grids were set up to register the velocities and the energies occurring at the end time. For each cell, the real and imaginary parts of the exponential were summed up. The probabilities for the various processors are calculated locally and then sent to the master. The average of the probabilities is calculated for every cell by the master. The decrease of the standard deviation can be controlled by the master processor at synchronisation points. For the numerical solution of the spatial distribution functions, every processor receives a space cell. The end points of all the generated paths on one processor are kept at this value. The occurring momentum end points are registered, as well as the energies and the velocities. Apart from the scattering potential, the approximation error depends on the discretization of the time interval and on the sizes of the cells.

\section{Results and Conclusions}

We calculate the energy, velocity, and wave vector distribution as a function of time. We use a continuous parameterization for the non-parabolic three valley conduction band structure over the Brillouin zone. The imaginary scattering potential is derived from first-order scattering rates. In Figure 1, one can see the probability of an elecı,ron to be encountered in wave-vector states for different time intervals elapsed since. the field was turned on and it started to propagate. The results obtained from standard Monte Carlo technique are reproduced in their tendency, although not in their accuracy. The probability to be found in an upper L-valley increases with time, while the Gamma-valley probability decreases. Due to the size of $\hbar$ the method should be applied for an energy and time range that fulfill $\epsilon(\vec{p}) / \hbar \cdot\left(t_{a}-\right.$ $\left.t_{b}\right) \approx 1$ otherwise leading to large oscillations that consume a huge amount of cpu-time.

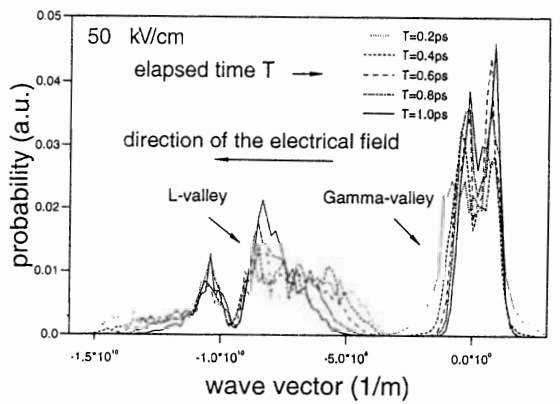

Figure 1: Momentum distribution function for bulk GaAs and constant electric field.

Acknowledgements: The author gratefully acknowledges the Deutsche Forschungsgemeinschaft for financial support.

\section{References}

[1] Massimo V. Fischetti and D. J. DiMaria, "Quantum Monte Carlo Simulation of High-Field Electron Transport: An Application to Silicon Dioxide," Phys. Rev. Lett., vol. 55, pp. 2475 - 2478, 1985.

[2] L. F. Register, M. A. Stroscio and M. A. Littlejohn, "Efficient path-integral Monte Carlo technique for ultrasmall device applications," Superlatt. M., vol. 6, pp. 233 - 243, 1989.

[3] Hagen Kleinert, "Pfadintegrals in Quantum Mechanics, Statistics and Polymer Physics", BI-Wiss.-Verl., 1993

[4] Malvin H. Kalos and Paula A. Whitlock, "Monte Carlo Methods", Wiley, 1986

[5] Dimitri P. Bertsekas and John N. Tsitsiklis, "Parallel and Distributed Computation", Prentice-Hall, 1989 